\title{
Transcatheter aortic valve replacement in intermediate and low risk patients-clinical evidence
}

\author{
Sameer Arora, John P. Vavalle \\ Division of Cardiology, University of North Carolina School of Medicine, Chapel Hill, NC, USA \\ Correspondence to: Sameer Arora, MD. Division of Cardiology, University of North Carolina, Chapel Hill, NC 27599, USA. Email: saror@email.unc.edu.
}

\begin{abstract}
The encouraging results of the PARTNER 2 (Placement of AoRtic TraNscathetER Valves 2) trial led to the approval of transcatheter aortic valve replacement (TAVR) in intermediate-surgical-risk patients. Recently, the SURTAVI (SUrgical Replacement and Transcatheter Aortic Valve Implantation) investigators demonstrated the feasibility of TAVR with self-expanding valves in intermediate-risk patients. The focus has now shifted to clinical trials comparing TAVR to surgery in low-surgical-risk populations with a goal to expand TAVR to all-risk patients. However, low-surgical-risk patients continue to be acceptable candidates for surgical aortic valve replacement, with proven outcomes over many decades. Although new data has emerged showing feasibility of TAVR in young patients with bicuspid valves, with newer generation TAVR valves there will be minimal tolerance for adverse outcomes in the low risk category. To expand the reach of TAVR into low-surgical-risk patients, important questions about valve durability, leaflet thrombosis, higher rates of paravalvular leak and permanent pacemakers (PPM) will need to be addressed. However, as TAVR technology continues to evolve, it seems to be just a matter of time before TAVR establishes itself as a modality for aortic valve replacement regardless of surgical risk.
\end{abstract}

Keywords: Transcatheter aortic valve replacement (TAVR); transcatheter aortic valve implantation (TAVI); transcatheter; low risk; intermediate risk

Submitted Jun 06, 2017. Accepted for publication Jun 13, 2017.

doi: $10.21037 /$ acs.2017.07.01

View this article at: http://dx.doi.org/10.21037/acs.2017.07.01

The original PARTNER (Placement of AoRtic TraNscathetER Valves) trial, conducted across multiple centers in the United States and Canada, led to the recognition of transcatheter aortic valve replacement (TAVR) as a feasible alternative to surgery in patients at high-surgical-risk and as the procedure of choice in those with inoperable-surgical-risk and a reasonable life expectancy $(1,2)$. The CoreValve US Pivotal trial, for the first time, demonstrated the superiority of TAVR with the self-expandable valve in comparison to surgery in highsurgical-risk patients (3). As a natural shift to broaden the indication to lower-surgical-risk patients, the intermediatesurgical-risk trials were approved comparing TAVR to surgery; both with the balloon-expandable SAPIEN XT valve (PARTNER 2 trial) and the self-expandable CoreValve [SUrgical Replacement and Transcatheter Aortic Valve Implantation trial (SURTAVI trial)] (4,5).
The PARTNER 2 trial was a randomized control trial conducted across 57 centers in the US and Canada, which enrolled 2,032 patients with severe symptomatic aortic stenosis and intermediate-surgical-risk and randomized them in a 1:1 fashion across the TAVR arm and the surgical arm. The primary endpoint was death and neurological events at 2 years. At 2 years, event rates were $19.3 \%$ in the TAVR group and $21.1 \%$ in the surgery group, but this did not meet statistical significance. In the transfemoral cohort, TAVR resulted in a lower rate of death or disabling stroke with a statistically significant difference. In addition, TAVR was found to have a lower risk of bleeding complications, acute kidney injury, and atrial fibrillation. Surgery was associated with fewer rates of vascular complications and paravalvular regurgitation. Prior to the results of the PARTNER 2 trial being reported, a metaanalysis of intermediate-surgical-risk studies had shown 
comparable outcomes between TAVR and SAVR (6). As a result of the PARTNER 2 trial, a 2017 focused update of the valvular heart disease guidelines from the American Heart Association and American College of Cardiology recommended TAVR as an alternative to surgery in patients at intermediate-surgical-risk (7). The SURTAVI trial, studying the self-expanding CoreValve in intermediaterisk patients, was a randomized, multicenter control trial which recruited a total of 1,746 intermediate-surgicalrisk patients at 87 centers. The patients were randomized in a 1:1 ratio of those undergoing TAVR with the use of a self-expanding bioprosthesis (CoreValve 84\%; Evolut R $16 \%$ ) or surgery. The primary endpoint was a composite death or disabling stroke at 24 months. At 24 months, the primary endpoint was reached in $12.6 \%$ of TAVR patients and $14.0 \%$ in the surgery patients. The rates of stroke were numerically in favor of TAVR without reaching statistical significance. Surgery was associated with higher rates of acute kidney injury, atrial fibrillation and requirement of blood transfusions. TAVR was noted to have higher rates of paravalvular regurgitation and new permanent pacemaker (PPM) implantation. Although the rates of PPM implantation have been found to be lower with the newer Evolut R, SURTAVI investigators found similar rates of PPM implantation with both the CoreValve (25.5\%) and the Evolut R valve (26.7\%).

As we look forward to extending TAVR to lower-riskpatients, it is crucial that major complications like PPM implantation have rates that remains comparable to that of surgery. Traditionally, higher rates of PPM have been associated with the self-expandable valves, as compared to the balloon-expandable valves $(8,9)$. However, the newer generation balloon-expandable Sapien 3 valves appear to have a higher PPM implantation rates than the older generation Sapien valves but this rate continues to be less than that of the self-expanding valves (10). The Evolut $R$ valve, the newer generation self-expanding valve, has reported lower PPM implantation rates compared with the first generation CoreValve, however, definitive data from randomized trials are still lacking due to the low number of Evolut R valves implanted in the SURTAVI trial (11). In a recent study, Maeno et al. investigated potential predictors of PPM in patients implanted with a third generation balloon-expandable valve (12). They found pre-TAVR right bundle branch block, height of membranous septum, and calcification volume at the noncoronary cusp device-landing zone to be independent predictors of PPM implantation. The 5-year PPM implantation rates from the ADVANCE study of the CoreValve devices were reported to be as high as $33.7 \%$ (9). Importantly, patients who underwent PPM implantation did not have a higher mortality than those who did not undergo PPM implantation.

Younger patients with severe AS are more likely to have bicuspid aortic valves (13) - the efficacy of TAVR in these patients continues to remain a topic of debate. The association of bicuspid AS with its higher incidence of aortopathy can lead to higher rates of aortic dissection; the extreme and asymmetrical calcification noted with bicuspid valves can prevent adequate expansion of the valve frame, affecting valve hemodynamics leading to higher aortic valve gradients and more paravalvular leaks (14). However, a recent study has shown that TAVR in bicuspid valves may just be a matter of operator experience. The Bicuspid TAVR Registry, initiated in December 2013, is a multicenter, multinational (20 centers across North America, Europe and Asia-Pacific) observational study which is investigating all patients with bicuspid aortic valves undergoing TAVR at enrolled institutions (15). The outcomes in these patients were compared between early generation (Sapien XT and CoreValve) and late generation TAVR valves (Sapien 3 and Lotus). Overall, the outcomes were similar, except lower rates of paravalvular regurgitation resulting in higher device success being noted for the newer generation valves.

Large scale clinical trials involving both the Medtronic CoreValve Evolut R System (NCT02701283) and the Edwards S3 system (PARTNER 3 trial, NCT02675114) have been approved in the low-surgical-risk category. The NOTION (Nordic Aortic Valve Intervention) trial, an all-comers trial with $81 \%$ low-surgical-risk patients is a multicenter, randomized, nonblinded superiority trial conducted in two centers in Sweden and one center in Denmark (16). A total of 280 patients underwent randomization between TAVR using the self-expanding prosthesis and surgery. The primary outcome of death due to any cause, stroke and myocardial infarction at 1 -year were similar in the two groups. The TAVR group had lower rates of bleeding, acute kidney injury, new or worsening atrial fibrillation or cardiogenic shock, a shorter length of stay and larger orifice areas. However, surgery had lower rates of pacemaker implantations and paravalvular regurgitation, and had a better New York Heart Association Class at 1 year. Additionally, a small meta-analysis found comparable short-term outcomes when comparing TAVR and surgery in patients with lower surgical risk scores (17).

As low-surgical-risk patients are expected to survive longer after TAVR when compared to higher-risk patients, 
the broad application of TAVR in low-risk patients should be limited until in vivo durability results are available for the TAVR prostheses (18). While structural valve deterioration in surgically-replaced valves has been thoroughly investigated $(19,20)$, long-term follow-up data for TAVR valves implanted in patients remains sparse. The 5 -year results from the PARTNER-1 trial, both for the highrisk study and the inoperable-risk study, did not show any evidence of structural deterioration of the TAVR valve at 5 years $(21,22)$.

However, data from St Paul's Hospital and Hôpital Charles Nicolle in Rouen, France, showed higher levels of degeneration for the balloon-expandable valve (23). A 50\% degeneration rate was noted at 8 years of follow-up, with the median degeneration time noted to be 61 months. The investigators defined valve degeneration as at least moderate regurgitation, or an aortic valve gradient $\geq 20 \mathrm{mmHg}$ in patients considered to have successful TAVR 30 days after implantation, a criterion which was debated rigorously. However, the role of internal mechanical pressure associated with balloon-expansion was hypothesized as one of the possible hypotheses for deterioration of the Sapien valves (24).

The durability data for the CoreValve was recently reported from the Italian investigators who were among the first ones to use the valve commercially (25). Patients in the registry have been enrolled from September 2007 and includes a total of 2,343 patients who have undergone TAVR. The overall mortality at 9-year follow-up was noted to be $73.5 \%$. They observed a total of 38 cases of valve dysfunction with clinical relevance, out of which 23 had led to fatality. A total of 27 cases were noted between the procedure and 1-year follow-up, out of which 7 were attributed to endocarditis and 20 due to degeneration of the leaflets leading to severe aortic regurgitation or stenosis. From the pool of patients who did not suffer from bioprosthesis dysfunction, a significant reduction in valve area or worsening of grade 3 paravalvular leak was not noted and the mean aortic gradient remained constant over follow-up. Recently, the NOTION trial investigators presented the 5-year data, making it the only study with a long-term follow up data on "real world" low-surgical-risk patients (26). The investigators defined durability as either bioprosthetic valve dysfunction or valve failure. Bioprosthetic valve dysfunction was categorized into structural valve deterioration, nonstructural valve deterioration, valve thrombosis or endocarditis. Valve failure was defined as valve-related death, aortic valve re-intervention or severe hemodynamic structural valve deterioration. Both bioprosthetic valve dysfunction and valve failure were similar between TAVR and surgery. However, structural valve deterioration was noted to be substantially higher in the surgery group. No cases of thrombosis were noted in either group. The results of this study put TAVR in a good light as far as its potential role for the treatment of low-surgical-risk patients.

Recent concerns regarding leaflet immobility caused by subclinical leaflet thrombosis have gained attention. The concept of leaflet immobility was first noticed during the PORTICO IDE trial (27). Close scrutiny including multiple CT images of a patient who suffered stroke following TAVR and another asymptomatic patient revealed leaflet thrombosis which was leading to reduced leaflet motion. This led to the formation of two physiciandirected registries RESOLVE and SAVORY (28). The objective of these registries was to investigate the prevalence of subclinical leaflet thrombosis in surgical and transcatheter aortic valves and to evaluate the efficacy of anticoagulants in treating the subclinical leaflet thrombosis, with the subsequent effect on valve haemodynamics and clinical outcomes. A total of 931 patients had CT imaging done as part of the two registries, out of which 890 had interpretable CT scans which showed 106 (12\%) cases of subclinical leaflet thrombosis. Out of 106, 5 (4\%) were in the SAVR group and $101(13 \%)$ were in the TAVR group $(\mathrm{P}=0.001)$. Only 8 out of 224 patients receiving anticoagulants had subclinical leaflet thrombosis versus $31(15 \%)$ out of 208 receiving dual antiplatelet therapy. Subclinical leaflet thrombosis persisted in 20 (91\%) of 22 patients not receiving anticoagulants, whereas it resolved in all 36 patients receiving anticoagulants $(\mathrm{P}<0.0001)$. A greater proportion of patients with subclinical leaflet thrombosis had aortic valve gradients of more than $20 \mathrm{mmHg}$ and increases in aortic valve gradients of more than $10 \mathrm{mmHg}$ [12 of $88(14 \%)$ ] than did those with normal leaflet motion [7 of 632 (1\%); $\mathrm{P}<0.0001$ ]. Subclinical leaflet thrombosis did not lead to higher stroke rates, however, increased rates of transient ischemic attacks were noted. These findings support the need to move judiciously into the low-risk space and to reserve doing so until randomized data with long-term follow-up is available. The ARTE trial recruited 222 patients and randomized them to receive aspirin versus dual antiplatelet therapy (aspirin plus clopidogrel) after TAVR (29). At 3 months, the rates of bleeding were significantly higher for the dual antiplatelet group without a significant benefit in terms of MI, stroke 
or death. Other trials, such as GALILEO (NCT02556203), which are comparing rivaroxaban with antiplatelet-based strategy in patients follow successful TAVR for prevention of leaflet thickening and reduced leaflet motion, will shed more light on this issue (30). Additionally, the FDA has now required all TAVR trials in the US to include a CT substudy to investigate leaflet mobility after TAVR. These data will prove invaluable as we learn about the incidence of valve thrombosis, leaflet immobility, and the role that anticoagulants and anti-platelet therapy has in reducing that risk.

As we have watched the rapid expansion of TAVR into lower surgical risk patients, we need to remain cautious about unbridled expansion into low-surgical risk patients, as many questions remain about valve durability, leaflet thrombosis, and higher rates of paravalvular leak and PPMs. The low-surgical-risk patients will continue to be acceptable candidates for surgery, with proven outcomes over many decades. However, there is little doubt that as TAVR technology continues to evolve and improve, many of the current hurdles described above will be overcome and TAVR will likely be the dominant modality for aortic valve replacement regardless of surgical risk.

\section{Acknowledgements}

None.

\section{Footnote}

Conflicts of Interest: The authors have no conflicts of interest to declare.

\section{References}

1. Smith CR, Leon MB, Mack MJ, et al. Transcatheter versus surgical aortic-valve replacement in high-risk patients. $\mathrm{N}$ Engl J Med 2011;364:2187-98.

2. Leon MB, Smith CR, Mack M, et al. Transcatheter aortic-valve implantation for aortic stenosis in patients who cannot undergo surgery. N Engl J Med 2010;363:1597-607.

3. Adams DH, Popma JJ, Reardon MJ. Transcatheter aorticvalve replacement with a self-expanding prosthesis. $\mathrm{N}$ Engl J Med 2014;371:967-8.

4. Leon MB, Smith CR, Mack MJ, et al. Transcatheter or Surgical Aortic-Valve Replacement in Intermediate-Risk Patients. N Engl J Med 2016;374:1609-20.
5. Reardon MJ, Van Mieghem NM, Popma JJ, et al. Surgical or Transcatheter Aortic-Valve Replacement in Intermediate-Risk Patients. N Engl J Med 2017;376:1321-31.

6. Arora S, Misenheimer JA, Jones W, et al. Transcatheter versus surgical aortic valve replacement in intermediate risk patients: a meta-analysis. Cardiovasc Diagn Ther 2016;6:241-9.

7. Nishimura RA, Otto CM, Bonow RO, et al. 2017 AHA/ ACC Focused Update of the 2014 AHA/ACC Guideline for the Management of Patients With Valvular Heart Disease: A Report of the American College of Cardiology/ American Heart Association Task Force on Clinical Practice Guidelines. J Am Coll Cardiol 2017;70:252-89.

8. Siontis GC, Jüni P, Pilgrim T, et al. Predictors of permanent pacemaker implantation in patients with severe aortic stenosis undergoing TAVR: a meta-analysis. J Am Coll Cardiol 2014;64:129-40.

9. Gerckens U, Tamburino C, Bleiziffer S, et al. Final 5-year clinical and echocardiographic results for treatment of severe aortic stenosis with a self-expanding bioprosthesis from the ADVANCE Study. Eur Heart J 2017. [Epub ahead of print].

10. Thourani VH, Kodali S, Makkar RR, et al. Transcatheter aortic valve replacement versus surgical valve replacement in intermediate-risk patients: a propensity score analysis. Lancet 2016;387:2218-25.

11. Popma JJ, Reardon MJ, Khabbaz K, et al. Early Clinical Outcomes After Transcatheter Aortic Valve Replacement Using a Novel Self-Expanding Bioprosthesis in Patients With Severe Aortic Stenosis Who Are Suboptimal for Surgery: Results of the Evolut R U.S. Study. JACC Cardiovasc Interv 2017;10:268-75.

12. Maeno Y, Abramowitz Y, Kawamori H, et al. A Highly Predictive Risk Model for Pacemaker Implantation After TAVR. JACC Cardiovasc Imaging 2017. [Epub ahead of print].

13. Pomerance A. Pathogenesis of aortic stenosis and its relation to age. Br Heart J 1972;34:569-74.

14. Makkar R, Chakravarty T, Jilaihawi H. Transcatheter Aortic Valve Replacement for Bicuspid Aortic Stenosis: Are We Ready for the Challenge? J Am Coll Cardiol 2016;68:1206-8.

15. Yoon SH, Lefèvre T, Ahn JM, et al. Transcatheter Aortic Valve Replacement With Early- and New-Generation Devices in Bicuspid Aortic Valve Stenosis. J Am Coll Cardiol 2016;68:1195-205.

16. Thyregod HG, Steinbrüchel DA, Ihlemann N, et al. 
Transcatheter Versus Surgical Aortic Valve Replacement in Patients With Severe Aortic Valve Stenosis: 1-Year Results From the All-Comers NOTION Randomized Clinical Trial. J Am Coll Cardiol 2015;65:2184-94.

17. Arora S, Strassle PD, Ramm CJ, et al. Transcatheter Versus Surgical Aortic Valve Replacement in Patients With Lower Surgical Risk Scores: A Systematic Review and Meta-Analysis of Early Outcomes. Heart Lung Circ 2017;26:840-5.

18. Arora S, Ramm CJ, Strassle PD, et al. Review of Major Registries and Clinical Trials of Late Outcomes After Transcatheter Aortic Valve Replacement. Am J Cardiol 2017;120:331-6.

19. Joshi V, Prosser K, Richens D. Early prosthetic valve degeneration with Mitroflow aortic valves: determination of incidence and risk factors $†$. Interact Cardiovasc Thorac Surg 2014;19:36-40.

20. Johnston DR, Soltesz EG, Vakil N, et al. Long-term durability of bioprosthetic aortic valves: implications from 12,569 implants. Ann Thorac Surg 2015;99:1239-47.

21. Mack MJ, Leon MB, Smith CR, et al. 5-year outcomes of transcatheter aortic valve replacement or surgical aortic valve replacement for high surgical risk patients with aortic stenosis (PARTNER 1): a randomised controlled trial. Lancet 2015;385:2477-84.

22. Kapadia SR, Leon MB, Makkar RR, et al. 5-year outcomes of transcatheter aortic valve replacement compared with standard treatment for patients with inoperable aortic stenosis (PARTNER 1): a randomised controlled trial. Lancet 2015;385:2485-91.

23. Arora S, Ramm CJ, Misenheimer JA, et al. Early transcatheter valve prosthesis degeneration and future ramifications. Cardiovasc Diagn Ther 2017;7:1-3.

24. Dvir D. First look at long-term durability of transcatheter

Cite this article as: Arora S, Vavalle JP. Transcatheter aortic valve replacement in intermediate and low risk patients-clinical evidence. Ann Cardiothorac Surg 2017;6(5):493-497. doi: 10.21037/acs.2017.07.01 heart valves: assessment of valve function up to 10 years after implantation. Available online: http://www.crtonline. org/presentation-detail/first-look-at-long-term-durabilityof-transcathete

25. Testa L. Prosthesis-related events and echocardiographic data throughout 9 years follow up after TAVI. Available online: http://www.crtonline.org/presentation-detail/ prosthesis-related-events-echocardiographic-data-t

26. Søndergaard L. Longevity of transcatheter and surgical bioprosthetic aortic valves in patients with severe aortic stenosis and lower surgical risk. Available online: https:// www.tctmd.com/slide/longevity-transcatheter-andsurgical-bioprosthetic-aortic-valves

27. Makkar RR, Fontana G, Søndergaard L. Possible Subclinical Leaflet Thrombosis in Bioprosthetic Aortic Valves. N Engl J Med 2016;374:1591-2.

28. Chakravarty T, Søndergaard L, Friedman J, et al. Subclinical leaflet thrombosis in surgical and transcatheter bioprosthetic aortic valves: an observational study. Lancet 2017;389:2383-92.

29. Rodés-Cabau J, Masson JB, Welsh RC, et al. Aspirin Versus Aspirin Plus Clopidogrel as Antithrombotic Treatment Following Transcatheter Aortic Valve Replacement With a Balloon-Expandable Valve: The ARTE (Aspirin Versus Aspirin + Clopidogrel Following Transcatheter Aortic Valve Implantation) Randomized Clinical Trial. JACC Cardiovasc Interv 2017;10:1357-65.

30. Comparison of a Rivaroxaban-based Strategy With an Antiplatelet-based Strategy Following Successful TAVR for the Prevention of Leaflet Thickening and Reduced Leaflet Motion as Evaluated by Four-dimensional, Volume-rendered Computed Tomography (4DCT) (GALILEO-4D). Available online: https://clinicaltrials. gov/ct2/show/NCT02833948 